\title{
Evaluación Anatómica del Tronco Celíaco y sus Ramas en una Muestra de Población Mestiza Colombiana
}

\author{
Anatomical Evaluation of the Celiac Trunk and its Branches \\ in a Sample of Colombian Mestizo Population
}

Bladimir Saldarriaga Tellez ${ }^{1,2}$ \& Luis Ernesto Ballesteros Acuña ${ }^{1,2}$

\begin{abstract}
SALDARRIAGA, T. B. \& BALLESTEROS, A. L. E. Evaluación anatómica del tronco celíaco y sus ramas en una muestra de población mestiza colombiana. Int. J. Morphol., 39(6):1743-1748, 2021.

RESUMEN: El tronco celíaco (TC) es la rama de la arteria aorta abdominal (AA) que aporta la irrigación a la porción distal del esófago, parte media del duodeno, al estómago, páncreas, bazo y suple adicionalmente al hígado; sus diferentes expresiones anatómicas son reportadas en los diferentes grupos poblacionales con incidencia variable. Se evaluó las características morfológicas del TC y sus ramas en 26 bloques del piso supramesocólico de cadáveres masculinos adultos no reclamados, del grupo poblacional mestizo, a quienes se les practico autopsia en el Instituto de Medicina Legal de Bucaramanga - Colombia. Se observo el tipo I del TC en 23 especímenes (88,4 \%), del cual correspondió 16 muestras $(61,5 \%)$. Al subtipo Ia con bifurcación y formación de tronco hepatoesplénico. Hubo un caso (3,8 \%) en donde las ramas del T se originaron de manera independiente de la AA. El TC presentó una longitud promedio de 18,6 DE 7,53 mm y un diámetro externo de $7 \mathrm{DE}$ 1,24 mm. De las ramas del TC, la AE presentó un diámetro promedio de 5,89 DE 1,04 mm sin diferencias estadísticamente significativa con relación al diámetro de la AHC, pero si con relación al diámetro de la AGI ( $\mathrm{P}=0,70 ; \mathrm{p}<0,001$ respectivamente). La gran mayoría de la muestra avaluada muestra la presencia de tronco hepatoesplénico seguido de la trifurcación en una verdadera configuración de trípode. El conocimiento de los patrones de ramificación del TC debe ser tomado en cuenta por cirujanos gastroenterólogos, radiólogos intervencionistas y oncólogos para evitar complicaciones durante los procedimientos quirúrgicos abdominales.
\end{abstract}

PALABRAS CLAVE: Tronco celíaco; Arteria hepática común; Arteria gástrica izquierda; Arteria esplénica, Variaciones anatómicas.

\section{INTRODUCCIÓN}

El tronco celíaco (TC) es la rama de la arteria aorta abdominal (AA) que aporta la irrigación a la porción distal del esófago,parte media del duodeno, al estómago, páncreas, bazo y suple adicionalmente al hígado.Clásicamente se describe de una manera general ramificándose en arteria hepática común (AHC), arteria esplénica (AE) y arteria gástrica izquierda (AGI). Este patrón trifurcado, denominado (tríada de Haller) ha sido descrito en un rango de 60,8 \% -95,5\% (Puelma \& Olave, 2010; Panagouli et al., 2013).

La expresión variante relacionada con la emergencia de las ramas del TC que se ha reportado con mayor incidencia tanto en estudios con material cadavérico como imagenológico en un rango de $8,2-76 \%$ es el tipo hepatoesplénico, en donde la AGI emerge proximalmente en el TC y luego se bifurca en AHC y AE (Petrella et al.,
2007; Malnar. et al., 2010; Prakash et al., 2012; Araujo Neto et al., 2015; Marco-Clement et al., 2016). Con baja incidencia (3,3 -20\%) se reporta la variante en donde del TC además de las ramas usuales, emergen como una o dos ramas adicionales las arterias frénica inferior, gastroduodenal y cólica media (Ragupathi \& Kumar, 2021).

La longitud del TC es de 7-29 mm (Malnar et al.; Seeben et al., 2013; Petrella et al.; Sehgal et al., 2013; Araujo Neto et al.). En tanto que su diámetro se ha reportado en un rango de 4-10 mm (da Silveira et al., 2009; Sehgal et al.; Sebben et al., 2013). Dada su importancia en el campo quirúrgico y en la interpretación imagenológica de las arterias que irrigan los órganos abdominales, diferentes estudios han reportado la distancia de la emergencia del TC a la emergencia de la arteria mesentérica superior (AMS). Esta distancia

\footnotetext{
${ }^{1}$ Profesor asociado Facultad de Salud. Universidad Autónoma de Bucaramanga, Colombia.

${ }^{2}$ Profesor titular Departamento de Ciencias Básicas Universidad Industrial de Santander, Colombia.

Proyecto financiado en la convocatoria bienal interna de investigaciones de la Universidad autónoma de Bucaramanga (UNAB) 2019-2020. Código 163.
} 
oscila en un amplio rango de 1-21,9 mm (Ekingen et al., 2021; Nigah et al., 2021; Ragupathi \& Kumar). Con relación a estos rasgos morfométricos, se ha presentado correlación significativa entre la longitud y diámetro del TC y entre el diámetro del TC y la distancia a la arteria AMS (Araujo Neto et al.).

La determinación de la morfología del TC ha sido descrita mediante técnicas clásicas de disección, abordajes quirúrgicos y estudios radiográficos, en otros grupos poblacionales distintos a la población mestiza latinoamericana, algunos con una clara orientación clínico-quirúrgica (Chen et al., 2009; Mburu et al., 2010; Prakash et al.; Song et al., 2010; Sehgal et al.; Ragupathi \& Kumar). La importancia del conocimiento de la expresión anatómica del TC y sus ramas, radica, además del concepto académico, en su impacto en la cirugía abdominal programada y de urgencias, en la interpretación de los estudios de imagen y en el manejo de eventos clínicos en los que estas estructuras anatómicas pueden verse comprometidas (Ragupathi \& Kumar). Teniendo en cuenta que el factor étnico es decisivo para la presencia de diferentes expresiones vasculares y la escasa información de este tipo en población mestiza, da relevancia al presente estudio, desarrollado en una muestra de material cadavérico colombiano, con el propósito de obtener información propia de referencia.

\section{MATERIAL Y MÉTODO}

Este estudio transversal, no probabilístico y descriptivo, determinó las características morfológicas del TC y sus ramas en 26 bloques del piso supramesocólico (hígado, estomago, páncreas, bazo, aorta abdominal y TC) de cadáveres masculinos adultos no reclamados, del grupo poblacional mestizo (descendientes de amerindios, blancos y negros), a quienes se les practicó autopsia en el Instituto de Medicina Legal de Bucaramanga - Colombia. Se consideró como criterios de exclusión el abordaje quirúrgico, trauma o las patologías que comprometieran a los órganos y estructuras vasculares contenidos en el piso supra meso cólico.

El muñón distal de la aorta abdominal y los orígenes de sus ramas emergentes lumbres, fueron anudados con seda 2/0 mientras que en el muñón proximal aórtico se instaló un catéter número 14 fijado con seda; los lechos vasculares fueron sometidos a lavado con solución salina fisiológica con el fin de eliminar los coágulos y restos hemáticos. Posteriormente, la AA, el TC y sus ramas se perfundieron a través del catéter instalado, con resina semisintética (mezcla de $85 \%$ de GP40L palatal con $15 \%$ de estireno y mineral de color rojo). La inyección de resina se detuvo al momento de verificarse presencia de presión positiva al nivel del émbolo de la jeringa utilizada para esta labor, lo que indicaba el pleno llenado de las estructuras arteriales a evaluar. Consecutivamente, los bloques abdominales fueron sometidos a fijación con formaldehido al $10 \%$ por 15 días.

Luego, el TC y sus ramas se disecaron desde su origen hasta sus segmentos distales. Posteriormente, se realizó medición con calibrador digital (mitutoyo $\left.{ }^{\circledR}\right)$, del diámetro externo del TC y de la AHC, AE y AGI a 0,5 mm de sus orígenes. Igualmente, se midió la longitud del TC y las distancia entre el origen de esta estructura vascular y el origen de la AMS.

Finalmente, se realizó la caracterización cualitativa de las diferentes expresiones morfológicas del TC y sus ramas emergentes, según los criterios de Marco-Clement et al.:

Tipo I: las ramas emergen del TCy pueden presentar una de las siguientes características.

a) Bifurcado, hepatoesplénico en el que la AGI emerge proximalmente (a un nivel más inferior) que las AHC y AE (Fig. 1).

b) Trifurcado(AHC, AE y AGI) emergen al mismo nivel (Fig. 2).

c) Tetrafurcado. Adicionalmente al patrón clásico emerge otra arteria.

Tipo II: TC bifurcado en el que una de sus ramas se origina independientemente de las otras; no se origina del TC si no de la AA. Pueden presentar una de las siguientes características.

a) TC hepatoesplénico. La AGI se origina independientemente de la AA.

b) TC gastroesplénico. La AHC se origina independientemente.

c) TC hepatogástrico. La AE se origina independientemente.

Tipo III: TC ausente las ramas se originan independientemente.

Tipo IV: TC mesentérico. En este se originan además de las ramas del TC, la AMS.

Se tomaron registros fotográficos a cada uno de los especímenes evaluados. Los estadísticos descriptivos, las representaciones gráficas y el contraste de hipótesis se realizaron con el software SPSS 20 (SPSS, Chicago, IL, EE. UU.) y Microsoft Excel 2013. Para cada parámetro morfométrico se realizó la prueba de normalidad de Kolmogórov-Smirnov, observándose una población normal para cada uno de estos parámetros. Las variables continuas se expresaron como promedios con intervalos de confianza del $95 \%$. El nivel considerado para indicar significancia estadística fue $\mathrm{p}<0,05$. Las variables cuantitativas siguieron una distribución normal y se utilizaron pruebas t para comparar los diferentes calibres entre arterias. 


\section{RESULTADOS Y DISCUSIÓN}

Se observó el tipo I del TC en 23 especímenes (88,4 $\%)$, del cual correspondió 16 muestras $(61,5 \%)$. al subtipo Ia con bifurcación y formación de tronco hepatoesplénico (Tabla I, Figs. 1 y 2).

El TC presento una longitud promedio de 18,6 DE $7,53 \mathrm{~mm}$ y un diámetro externo de 7DE 1,24 mm. Correspondiendo la mayor longitud al subtipo Ia con 21,8 DE 8,12

Tabla I. Frecuencia de los patrones de tronco celíaco (TC) según subtipos

\begin{tabular}{lll}
\hline Subtipo & Muestra & Porcentaje \\
\hline Ia & 16 & 61,5 \\
Ib & 7 & 26,9 \\
IIa & 1 & 3,8 \\
IIb & 1 & 3,8 \\
III & 1 & 3,8
\end{tabular}

Ia: T.C. hepatoesplénico, Ib: TC trifurcado, IIa: T.C. hepatoesplénico; Ilb: T.C. gastroesplénico, III: ausencia del TC.

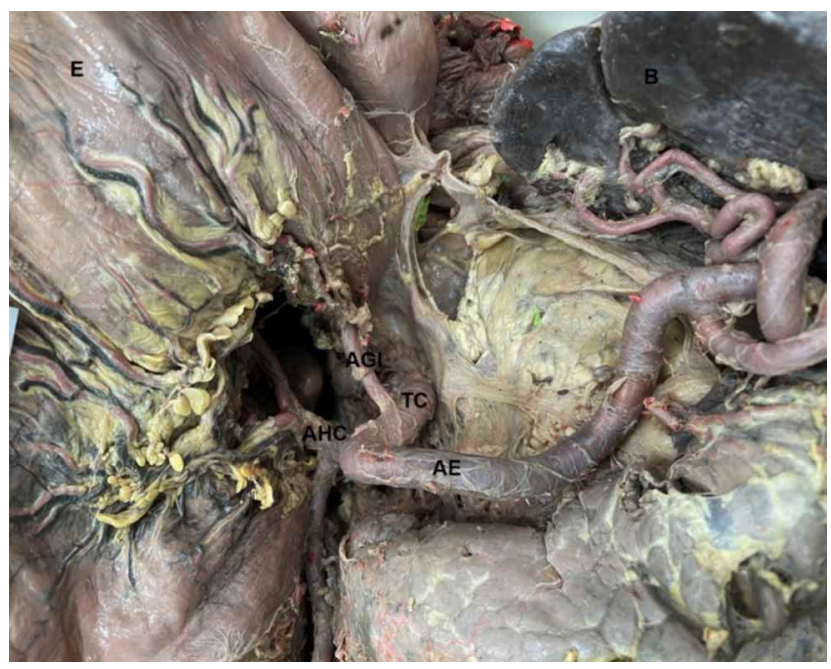

Fig. 1. Tronco celíaco hepatoesplénico (Ia). AE: arteria esplénica, AGI: arteria gástrica izquierda, AHC: arteria hepática común, B: bazo, E: estómago, TC: tronco celiaco mm. De las ramas del TC, la AE presento un diámetro promedio de 5,89 DE 1,04 mm sin diferencias estadísticamente significativa con relación al diámetro de la AHC, pero si con relación al diámetro de la AGI $(\mathrm{P}=0,70 ; \mathrm{p}<0,001$ respectivamente). La AMS se originó a una distancia del TC de 11,1 DE 4,44 mm. No hubo diferencias estadísticamente significativas entre la distancia de los subtipos del TC al origen de la AMS $(t=-0,985)$ (Tabla II).

Los trabajos que incluyen revisiones sistemáticas de la literatura en torno a la incidencia de las diferentes expresiones anatómicas del TC como los de Panagouli et al., señalan que el TC trifurcado se presenta en un rango de $54 \%$ - 98,3\%., incluyendo al trípode de Haller o cuando una de las arterias emerge proximalmente en el TC. En nuestro estudio incluyendo los subtipos Ia (hepatoesplénico), Ib (las ramas emergen al mismo nivel) la frecuencia fue de $88,4 \%$, que podemos considerar en la franja alta, concordantes con lo reportado por da Silveira et al. (85,7 \%), Prakash et al. (86\%), Son et al. $(89,1 \%)$ Chen et al. $(89,8 \%)$ y notoriamente superiores a lo reportado por Mburu et al. $(61,8 \%)$.

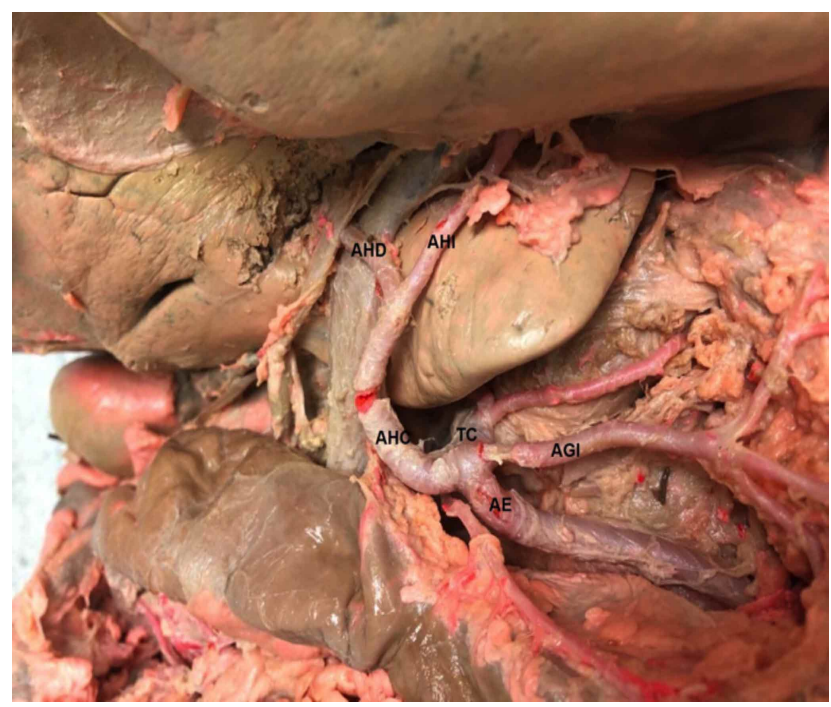

Fig. 2. Tronco celíaco trifurcado (hepatogastroesplénico). AE: arteria esplénica, AGI: arteria gástrica izquierda, AHC: arteria hepática común, AHD: arteria hepática derecha, AHÍ: arteria hepática izquierda, TC: tronco celiaco.

Tabla II. Morfometría de los subtipos del tronco celíaco (longitud y diámetro en milímetros) de sus ramas y distancia al origen de la arteria mesentérica superior.

\begin{tabular}{lrrccccc}
\hline S ubtipo & Long TC & D- TC & Dist. TC-AMS & Diam. AMS & Diam. AHC. & Diam. AGI & Diam. AE \\
\hline Ia & 21,8 & 7,2 & 11,9 & 8,9 & 5,4 & 3,7 & 6,1 \\
Ib & 15,6 & 6,2 & 7,6 & 5,5 & 5,3 & 4,8 & 5,8 \\
IIa & 21,0 & 6,8 & 13,2 & 7,2 & 4,6 & 5,6 & 5,6 \\
IIb & 5,9 & 7,9 & 13,5 & 7,6 & 4,2 & 5,7 & 5,59 \\
Total & 18,6 & 7 & 11,6 & 7,46 & 5,23 & 0,80 \\
DE & 7,53 & 1,25 & 4,4 & 1,86 & 1,23 & 1,04 \\
\hline
\end{tabular}

Long TC: Longitud TC, D-TC: Diametro TC, Dist TC-AMS: Distancia TC a AMS. Diam: Diametro, AHC: artera hepática común, AGI: arteria gástrica izquierda. AE: arteria esplénica, AMS: arteria mesentérica superior. 
Las variaciones anatómicas más frecuentes son las de TC bifurcado (Dos Santos et al., 2018). En nuestro estudio el subtipo hepatoesplénico en el que el TC se bifurca en AHC y AE y la AGI emerge proximalmente del TC fue el observado con mayor frecuencia $(61,5 \%)$ y es concordante con las cifrasreportadas por Clement et al., $(57,6 \%)$, Chen et al. $(66,6 \%)$ y considerablemente más altos a los reportados por Pinal-García et al. (2018) (32,1 \%), Puelma \& Olave $(37,8 \%)$. El subtipo $\mathrm{Ib}$, trifurcado hepatogastroesplénico en el que las tres ramas del TC emergen al mismo nivel (trípode de Haller), encontramos una frecuencia de 26,9\%. En la literatura se reportan frecuencias entre 19,4\% - $47 \%$ (Marco-Clement et al.; Pillay et al., 2020). Concuerdan nuestros datos con los reportados por Clement et al., 2016 (32,1\%). Superiores a los reportados, 7,1 \% (Pinal-García et al.) 22,2 $\%$ (Petrella et al.). E inferiores 60,8 \% (Puelma \& Olave), $47 \%$ (Pillay et al.), 89,5\% (Song et al.) y $90 \%$ (D'Souza \& Mamatha, 2012).

El tipo IIa, TC hepatoesplénico, en el que la AGI se origina independientemente de la AA, se reportan incidencias de $0-13 \%$ (Panagouli et al.; Marco-Clement et al.). La incidencia de este tipo encontrada en nuestra serie $(3,8 \%)$ es concordante por lo reportado en algunos estudios previos (Pillay et al.; Chen et al.; da Silveira et al.; Song et al.) y considerablemente más alta que la obtenida por Pinal-García et al. de 1,4\% en un estudio en 140 especímenes cadavéricos. Mientras que el TC tipo IIb (gastroesplénico) ha sido reportado por la mayoría de estudios previos con una incidencia de 2,3-5,5 \% (Marco-Clement et al.; Chen et al.; Song et

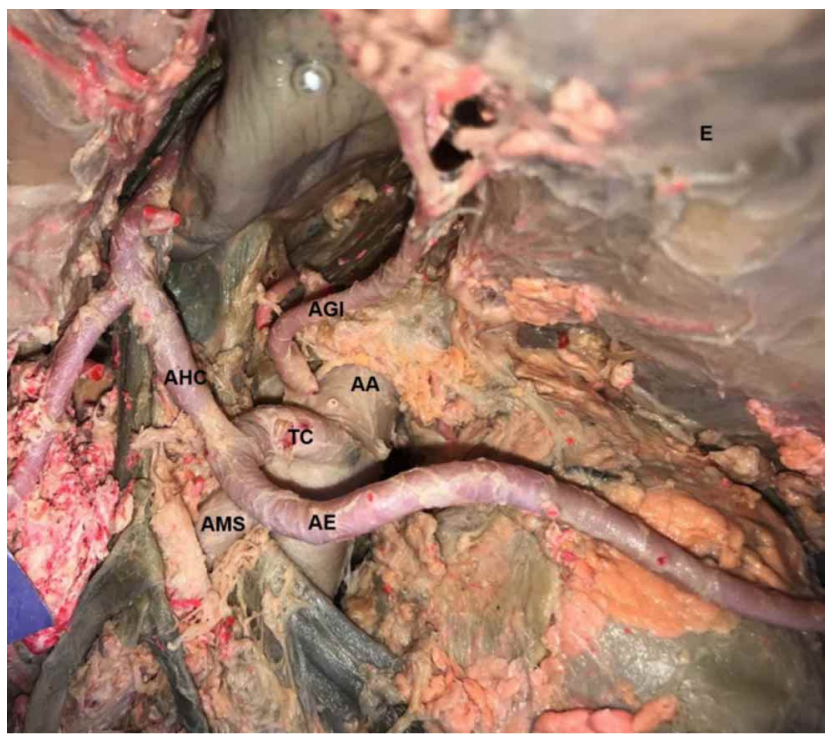

Fig. 3. Tronco celíaco hepatoesplénico. AA: arteria aorta abdominal, AE: arteria esplénica, AGI: arteria gástrica izquierda, AHC: arteria hepática común, AHD: arteria hepática derecha, AHÍ: arteria hepática izquierda, AMS: arteria mesentérica superior, TC: tronco celiaco. $a l$.$) , cifras que son concordantes con nuestros hallazgos. La$ más baja frecuencia de esta variedad anatómica $(1,5 \%)$ es reportada por Pillay et al.

La ausencia de TC (tipo III) se ha reportado en un rango de 0-4\%. Panagouli et al., en su revisión sistemática que incluyo 36 artículos, reportan una frecuencia promedio de $0,38 \%$. Nuestros resultados $(3.8 \%)$ se ubican en la franja alta concordante con lo reportado por Prakash et al. y Sehgal et al. La mayoría de estudios previos reportan esta expresión morfológica con incidencias menores al $2 \%$. (Petrella et al.).

El diámetro del TC encontrado en nuestra serie (7 $\mathrm{mm})$, es concordante con el reportado por Malnar et al. $(6,5$ $\mathrm{mm})$, Pinal-García et al. $(7,2 \mathrm{~mm})$ da Silveira et al. $(7,9$ $\mathrm{mm}$ ). Se destaca el calibre del TC de 13,3 $\mathrm{mm}$ reportado por Sebben et al. Algunos estudios previos reportan disminución del diámetro del TC cuando se presentan variaciones anatómicas (da Silveira et al.; Malnar et al.), característica que no fue observada en nuestro trabajo en el que las presentaciones variantes (Ia, IIa y IIb) tuvieron un mayor diámetro que el TC trifurcado.

La longitud promedio de los diferentes tipos de TC obtenidos en nuestro estudio $(18,6 \mathrm{~mm})$ se ubica en un nivel medio al contrastarla con reportes obtenidos de material cadavérico y angiográfico que señalan esta dimensiónen un rango de 6,8-35 mm (Sebben et al.; Petrella et al.; Malnar et al.; Sehgal et al.). La variabilidad en los reportes de la lon-

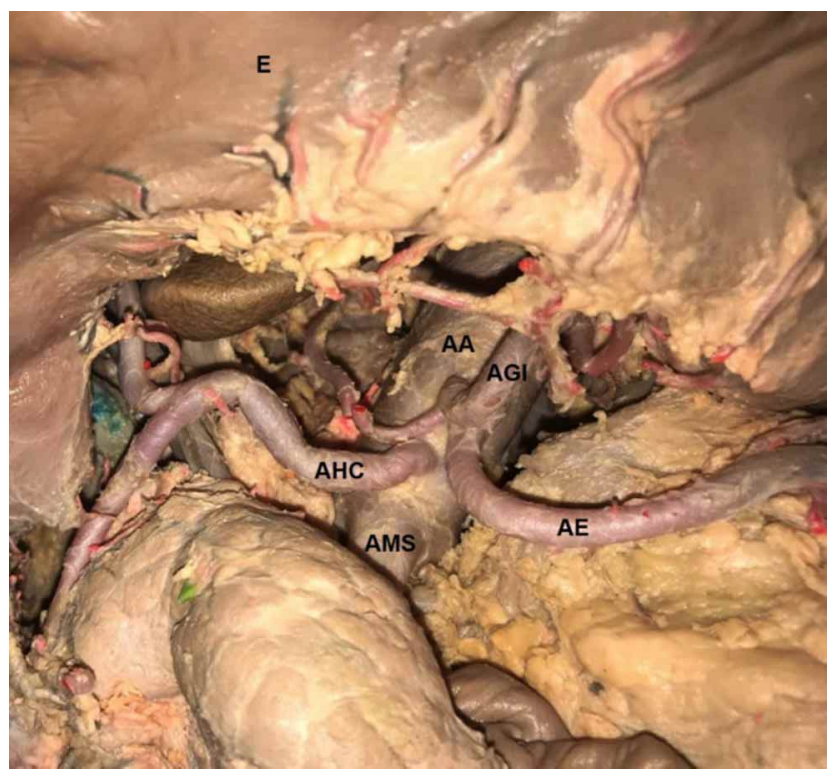

Fig. 4. Tronco celíaco ausente (tipo III). AA: arteria aorta abdominal, AE: arteria esplénica, AGI: arteria gástrica izquierda, AHC: arteria hepática común, AMS: arteria mesentérica superior, E: estómago, TC: tronco celiaco. 
gitud del TC puede atribuirse al tamaño de las muestras, las características biológicas de los grupos poblacionales estudiados y a los criterios de medición en cada uno de los estudios realizados, desde el origen hasta la emergencia de la primera o ultima rama.

Se ha reportado La distancia del TC a la AMS en un rango de 1-21 mm (Takahashi et al., 2013; Mazzaccaro et al., 2015; Nigah et al.; Ekingen et al.). Nuestros resultados $(11,6 \mathrm{~mm})$ son concordantes con lo reportado por Araujo Neto et al., Ekingen et al. y Nigah et al. Otros autores reportan distancias considerablemente mayores en un rango de 19,3-21,9 mm (Yadav et al., 2014; Ragupathi \& Kumar). Se destaca que, en nuestros hallazgos, el subtipo trifurcado, (Ib) presentó una distancia al origen de la AMS menor con relación a los demás subtipos, pero sin diferencia estadísticamente significativa $(\mathrm{t}=0,985)$. Esta inferencia estadística que valora la distancia del origen de la AMS y los subtipos del TC no se ha reportado en la mayoría de estudios previos (Mburu et al.; da Silveira et al.). Ragupathi \& Kumar señalan la inexistencia de diferencias significativas entre la distancia de estos vasos cuando se presentan variables anatómicas del TC.

La distancia entre los orígenes del TC y la AMS es un rasgo anatómico importante a tener en cuenta en los procedimientos que comprometan estas estructuras, dado que los orígenes de estos vasos se hallan rodeados de los ganglios y linfonodos celíaco y mesentérico superior, las intervenciones quirúrgicas, especialmente de urgencia, en pacientes en donde el TC y la AMS se hallan a una distancia menor a un centímetro, sumado a un campo de operación hemorrágico y edematizado, puede conducir a complicaciones iatrogénicas por ligadura o lesión de la inervación de las vísceras que se ubican en los sectores supra e inframesocólico de la cavidad abdominal (Kowalczyk \& Majewski, 2021).

La existencia de una extensa red anastomótica entre las arterias originadas del TC y la AMS hace que la estenosis del TC sea asintomática en la mayoría de los casos. Esta anastomosis puede ocurrir a través de ramas de las arterias pancreaticoduodenales superior e inferior en la cabeza del páncreas, o ramas de la arteria pancreática dorsal en el cuerpo del páncreas. La estenosis del TC se informa en aproximadamente uno de cada diez pacientes sometidos a pancreaticoduodenectomía realizadas para la extirpación de colangiocarcinomas y tumores ampulares (Thompson et al., 1981).

Dada la gran variabilidad en la expresión morfológica del TC y sus ramas, la información preoperatoria en torno a estas estructuras puede contribuir a la reducción del tiempo quirúrgico y las complicaciones vasculares perioperatorias como sangrado e isquemia, especialmente para el manejo de la aterosclerosis y aneurismas mediante procedimientos endovasculares así como la extirpación del lecho tumoral y de los linfonodos circundantes en las neoplasias malignas gastrointestinales En este sentido, conocer las variaciones vasculares durante el período preoperatorio permitirá el diseño de abordajes quirúrgicos individualizados para cada paciente en concordancia con la configuración individual del origen de estas arterias (Ekingen et al.).

\section{CONCLUSIONES}

La gran mayoría de la muestra avaluada muestran la presencia de tronco hepatoesplénico seguido de la trifurcación en una verdadera configuración de trípode. El haber encontrado menos variaciones a las reportadas en otros grupos poblacionales sugiere que un estudio futuro con una muestra más grande puede arrojar resultados más concluyentes que posibiliten hacer inferencias de estos rasgos anatómicos en la población colombiana. El conocimiento de los patrones de ramificación del TC debe ser tomado en cuenta por cirujanos gastroenterólogos, radiólogos intervencionistas y oncólogos para evitar complicaciones durante los procedimientos quirúrgicos y radiológicos abdominales.

\section{AGRADECIMIENTOS}

Al Instituto de Medicina Legal y Ciencias Forenses Regional Nor-Oriente por la donación de los bloques anatómicos objeto del presente estudio.

SALDARRIAGA, T. B. \& BALLESTEROS, A. L. E. Anatomical evaluation of the celiac trunk and its branches in a sample of Colombian mestizo population. Int. J. Morphol., 39(6):1743-1748, 2021.

SUMMARY: The celiac trunk (CT) is the branch of the abdominal aorta artery (AA) that provides irrigation to the distal portion of the esophagus, the middle part of the duodenum, the stomach, pancreas, spleen and additionally supplies the liver; its different anatomical expressions are reported in the different population groups with variable incidence. The morphological characteristics of the CT and its branches were evaluated in 26 blocks of the supramesocolic floor of unclaimed adult male corpses, of the mestizo population group, who were autopsied at the Institute of Legal Medicine of Bucaramanga - Colombia. Type I CT was observed in 23 specimens (88.4\%), of which 16 samples $(61.5 \%)$ corresponded. to subtype Ia with bifurcation and formation of the hepatosplenic trunk. There was one case $(3.8 \%)$ in which the branches of the CT originated independently of the AA. The CT 
had an average length of $18.6 \mathrm{SD} 7.53 \mathrm{~mm}$ and an external diameter of $7 \mathrm{SD} 1.24 \mathrm{~mm}$. Of the CT branches, the splenic artery presented an average diameter of $5.89 \mathrm{SD} 1.04 \mathrm{~mm}$ without statistically significant differences in relation to the diameter of the AHC, but if in relation to the diameter of the IGA $(\mathrm{P}=0.70 ; \mathrm{p}<0.001$ respectively). The vast majority of the sample evaluated shows the presence of a hepatosplenic trunk followed by trifurcation in a true tripod configuration. Knowledge of CT branching patterns should be taken into account by gastroenterological surgeons, interventional radiologists, and oncologists to avoid complications during abdominal surgical procedures.

KEY WORDS: Celiac trunk; Common hepatic artery; Left gastric artery; Splenic artery, Anatomical variations.

\section{REFERENCIAS BIBLIOGRÁFICAS}

Araujo Neto, S. A.; Franca, H. A.; de Mello Júnior, C. F.; Silva Neto, E. J.; Negromonte, G. R. P.; Duarte, C. M. A.; Cavalcanti Neto, B. F. \& da Fonseca Farias, R. D. Anatomical variations of the celiac trunk and hepatic arterial system: an analysis using multidetector computed tomography angiography. Radiol. Bras., 48(6):358-62, 2015.

Chen, H.; Yano, R.; Emura, S. \& Shoumura, S. Anatomic variation of the celiac trunk with special reference to hepatic artery patterns. Ann. Anat., 191(4):399-407, 2009.

D'Souza, A. S. \& Mamatha, H. Anatomical variations in the branches of the coeliac trunk. J. Clin. Diagn. Res., 6(3):333-5, 2012.

da Silveira, L. A.; Silveira, F. B. C. \& Fazan, V. P. S. Arterial diameter of the celiac trunk and its branches. Anatomical study. Acta Cir. Bras., 24(1):43-7, 2009

Dos Santos, P. V.; Barbosa, A. B. M.; Targino, V. A.; Silva, N. A.; Silva, Y. C.; Barbosa, F.; Oliveira, A. S. B. \& Assis, T. O. Anatomical variations of the celiac trunk: a systematic review. ABCD. Arq. Bras. Cir. Dig., 31(4):e1403, 2018.

Ekingen, A.; Hatipog $`$ lu, E. S. \& Hamidi, C. Distance measurements and origin levels of the coeliac trunk, superior mesenteric artery, and inferior mesenteric artery by multiple-detector computed tomography angiography. Anat. Sci. Int., 96(1):132-41, 2021.

Kowalczyk, K. A. \& Majewski, A. Analysis of surgical errors associated with anatomical variations clinically relevant in general surgery. Review of the literature. Transl. Res. Anat., 23:100107, 2021.

Malnar, D.; Klasan, G. S.; Miletic, D.; Bajek, S., Vranic, T. S.; Arbanas, J.; Bobinac, D. \& Coklo, M. Properties of the celiac trunk--anatomical study. Coll. Antropol., 34(3):917-21, 2010.

Marco-Clement, I.; Martinez-Barco, A.; Ahumada, N.; Simon, C.; Valderrama, J. M.; Sanudo, J. \& Arrazola, J. Anatomical variations of the celiac trunk: cadaveric and radiological study. Surg. Radiol. Anat., 38(4):501-10, 2016.

Mazzaccaro, D.; Malacrida, G. \& Nano, G. Variability of origin of splanchnic and renal vessels from the thoracoabdominal aorta. Eur. J. Vasc. Endovasc. Surg., 49(1):33-8, 2015.

Mburu, K. S.; Alexander, O. J.; Hassan, S. \& Bernard, N. Variations in the branching pattern of the celiac trunk in a Kenyan population. Int. J. Morphol., 28(1):199-204, 2010.

Nigah, S.; Patra, A.; Chumbar, S. \& Chaudhary, P. Topographic location and branching pattern of the superior mesenteric artery with its clinical relevance: a cadaveric study. Folia Morphol. (Warsz), 2021. DOI: https:/ /www.doi.org/10.5603/FM.a2021.0031

Panagouli, E.; Venieratos, D.; Lolis, E. \& Skandalakis, P. Variations in the anatomy of the celiac trunk: A systematic review and clinical implications. Ann. Anat., 195(6):501-11, 2013.
Petrella, S.; de Sousa Rodríguez, C. F.; Sgrott, E. A.; Fernandes, G. J. M.; Marques, S. R. \& Prates, J. C. Anatomy and variations of the celiac trunk. Int. J. Morphol., 25(2):249-57, 2007.

Pillay, D.; Nischk, S. D. \& Ndou, R. Variations in the configuration of the celiac trunk among South Africans: Clinical relevance. Int. J. Morphol., 38(6):1662-7, 2020

Pinal-García, D. F.; Nuno-Guzmán, C. M.; Gonzalez-Gonzalez, M. E. \& Ibarra-Hurtado, T. R. The celiac trunk and its anatomical variations: a cadaveric study. J. Clin. Med. Res., 10(4):321-9, 2018.

Prakash; Rajini, T.; Mokhasi, V.; Geethanjali, B. S.; Sivacharan, P. V. \& Shashirekha, M. Coeliac trunk and its branches: anatomical variations and clinical implications. Singapore Med. J., 53(5):329-31, 2012.

Puelma, F. \& Olave, E. Relationships between of the origin of celiac trunk, mesenteric and renal arteries with the vertebral column in Chilean individuals. Int. J. Morphol., 28(4):1227-34, 2010.

Ragupathi, S. \& Kumar, G. P. Clinical significance of celiac trunk and superior mesenteric artery diameter following computed tomography. Int. J. Anat. Radiol. Surg., 10(2):49-51 2021.

Sebben, G. A.; Rocha, S. L.; Sebben, M. A.; Parussolo Filho, P. R. \& Gonçalves, B. H. H. Variations of hepatic artery: anatomical study on cadavers. Rev. Col. Bras. Cir., 40(3):221-6, 2013.

Sehgal, G.; Srivastava, A. K.; Sharma, P. K.; Kumar, N.; Singh, R.; Parihar, A. \& Aga, P. Morphometry of the celiac trunk: a multidetector computed tomographic angiographic study. J. Anat. Soc. India, 62(1):23-7, 2013.

Song, S. Y.; Chung, J. W.; Yin, Y. H.; Jae, H. J.; Kim, H. C.; Jeon, U. B.; Cho, B. H.; So, Y. H. \& Park, J. H. Celiac axis and common hepatic artery variations in 5002 patients: systematic analysis with spiral CT and DSA. Radiology, 255(1):278-88, 2010.

Takahashi, T.; Takeuchi, K.; Ito, T. \& Itoh, M. Positional relationships among the celiac trunk, superior mesenteric artery, and renal artery observed from the intravascular space. Surg. Radiol. Anat., 35(5):411-7, 2013.

Thompson, N. W.; Eckhauser, F. E.; Talpos, G. \& Cho, K. J. Pancreaticoduodenectomy and celiac occlusive disease. Ann. Surg., 193(4):399-406, 1981.

Yadav, S. P.; Sinha, R. S. \& Patil, T. Study of variations of coeliac trunk in western Maharashtra population. Int. J. Curr. Res. Rev., 6(23):31-8, 2014

Dirección para correspondencia:

Bladimir Saldarriaga

T.7-6436111-ext-511

Calle $157-14-55$

Campus El Bosque

Floridablanca (Santander)

COLOMBIA

E-mail: vsaldarr@unab.edu.co

Recibido : 28-05-2021

Aceptado: 18-08-2021 